\title{
FACTOR GRAPH BASED DESIGN OF AN OFDM-IDMA RECEIVER PERFORMING JOINT DATA DETECTION, CHANNEL ESTIMATION, AND CHANNEL LENGTH SELECTION
}

\author{
Clemens Novak, Gerald Matz, and Franz Hlawatsch \\ Institute of Communications and Radio-Frequency Engineering, Vienna University of Technology \\ Gusshausstrasse 25/389, A-1040 Vienna, Austria; e-mail: clemens.novak@tuwien.ac.at
}

\begin{abstract}
We present a factor graph based design of a receiver for pilotassisted OFDM-IDMA systems transmitting over frequency-selective channels. The receiver performs joint iterative multiuser data detection and channel estimation with a complexity that is linear in the number of users, and it includes estimation of the channel length. Simulation results demonstrate large performance gains compared to OFDM-IDMA receivers using separate MMSE channel estimation.
\end{abstract}

Index Terms-OFDM-IDMA, multiuser detection, channel estimation, iterative receiver, factor graph, sum-product algorithm

\section{INTRODUCTION}

Multiuser systems employing interleave-division multiple access (IDMA) achieve user separation by means of user-specific interleavers combined with low-rate channel coding [1]. OFDM-IDMA was introduced in $[2,3]$ as an extension for frequency-selective channels.

In [4], we proposed a factor graph based design of an IDMA receiver performing joint data detection and pilot-assisted channel estimation. Here, we present a similar design for OFDM-IDMA systems transmitting over frequency-selective channels. While our development is largely analogous to [4], the frequency-selective channel model and OFDM modulation scheme result in different messages in parts of the factor graph. We also propose an explicit estimation of the length of the channel impulse response, thereby reducing the number of channel coefficients to be estimated. The entire receiver-performing joint multiuser data detection, channel estimation, and channel length selection-is derived by applying the sum-product algorithm [5] to the Forney-style factor graph [6] of the OFDM-IDMA system (cf. [7, 8]). A complexity that is linear in the number of users is achieved by Gaussian message approximations.

This paper is organized as follows. The OFDM-IDMA system is described in Section 2. The factor graph and messages are developed in Sections 3 and 4, respectively. Section 5 discusses channel length estimation. Simulation results are provided in Section 6.

\section{PILOT-ASSISTED OFDM-IDMA SYSTEM}

We consider a synchronous uplink scenario where $U$ users transmit data to a base station via OFDM-IDMA transmitters (see Fig. 1). The bits of the $u$ th user, $\mathbf{b}^{u} \triangleq\left(b_{1}^{u} \cdots b_{K}^{u}\right)^{T}$, are converted into a length$M$ BPSK symbol vector $\mathbf{x}^{u}=\mathcal{C}^{u}\left(\mathbf{b}^{u}\right)$ by means of a terminated convolutional code serially concatenated with a low-rate repetition code, a user-specific interleaver $\pi^{u}(\cdot)$, and a symbol mapper. Each of the $M$ BPSK data symbols is transmitted on a corresponding OFDM subcarrier; we thus write the data symbols as $x_{n}^{u}$, where $n$ denotes

This work was supported by the STREP project MASCOT (IST-026905) within the Sixth Framework of the EC and by the FWF projects "Statistical Inference" (S10603-N13) and "Information Networks" (S10606-N13) within the National Research Network SISE.

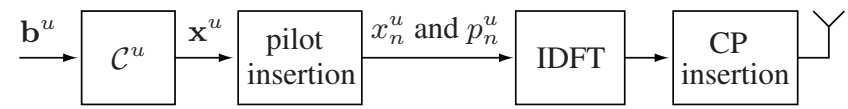

Fig. 1. OFDM-IDMA transmitter for the $u$ th user.

the subcarrier index. The transmitter uses a total of $N=M+U N_{p}$ subcarriers, consisting of $M$ data subcarriers (allocated jointly to all users) and $U N_{p}$ pilot subcarriers. The $u$ th user transmits $N_{p}$ pilot symbols $p_{n}^{u}$ on $N_{p}$ user-specific pilot subcarriers spaced $\Delta$ subcarriers apart. Let $\mathcal{P}^{u}$ denote the corresponding set of pilot subcarriers and $\mathcal{P}$ the union of all the (disjoint) sets $\mathcal{P}^{u}$. The subcarriers $n=1, \ldots, N$ are partitioned into $N_{p}$ blocks of $\Delta$ subcarriers consisting of $U$ pilot subcarriers (one for each user) and $\Delta-U$ data subcarriers. Thus, $N_{p}(\Delta-U)=M$ and therefore $N_{p}=\left|\mathcal{P}^{u}\right|=\frac{M}{\Delta-U}$. Finally, the time-domain transmit signal of each user is obtained by applying an inverse discrete Fourier transform (IDFT) to the user's data and pilot symbols and inserting a cyclic prefix.

The channel of the $u$ th user is frequency-selective with time-domain impulse response denoted by the length- $N$ vector $\tilde{\mathbf{h}}^{u} \triangleq\left(\tilde{h}_{1}^{u} \ldots\right.$ $\left.\tilde{h}_{L_{c}}^{u} 0 \cdots 0\right)^{T}$. The $L_{c}$ nonzero channel taps are assumed uncorrelated and zero-mean complex Gaussian, i.e., $\tilde{\mathbf{h}}^{u} \sim \mathcal{C N}\left(\mathbf{0}, \mathbf{C}_{\tilde{h}_{p}}\right)$, where $\mathbf{C}_{\tilde{h}_{p}}$ (subscript $p$ stands for "prior") is a diagonal $N \times N$ matrix in which only the first $L_{c}$ diagonal elements are nonzero. The frequency-domain channel coefficient vector is given by $\mathbf{h}^{u} \triangleq$ $\left(h_{1}^{u} \cdots h_{N}^{u}\right)^{T}=\mathbf{F} \tilde{\mathbf{h}}^{u}$, where $\mathbf{F}$ is the unitary $N \times N$ DFT matrix.

At the receiver, the cyclic prefix is removed, and a DFT yields the frequency-domain receive vector $\mathbf{r}$ of length $N$. This vector consists of $M$ entries involving the data symbols $x_{n}^{u}$ of all users,

$$
r_{n}=\sum_{u=1}^{U} h_{n}^{u} x_{n}^{u}+w_{n}, \quad n \notin \mathcal{P},
$$

and $U N_{p}$ entries (denoted $\tilde{r}_{n}^{u}$ ) corresponding to the pilot symbols,

$$
\tilde{r}_{n}^{u}=h_{n}^{u} p_{n}^{u}+w_{n}, \quad n \in \mathcal{P}^{u}, \quad u=1, \ldots, U .
$$

Here, $w_{n}$ denotes white Gaussian noise of variance $\sigma_{w}^{2}$.

\section{FACTOR GRAPH AND RECEIVER STRUCTURE}

The proposed receiver is based on the bitwise MAP detector [7]

$$
\hat{b}_{i}^{u}=\arg \max _{b_{i}^{u} \in\{0,1\}} p\left(b_{i}^{u} \mid \mathbf{r}\right),
$$

where $p\left(b_{i}^{u} \mid \mathbf{r}\right)$ is the posterior probability mass function of bit $b_{i}^{u}$.

Factor graph. The marginalization involved in (3) can be done efficiently by applying the sum-product algorithm to the factor graph of the OFDM-IDMA system [5,7,8]. Let $\mathbf{b}$ denote the vector of all $b_{i}^{u}$. For equally likely $b_{i}^{u}$, we obtain from Bayes' rule 


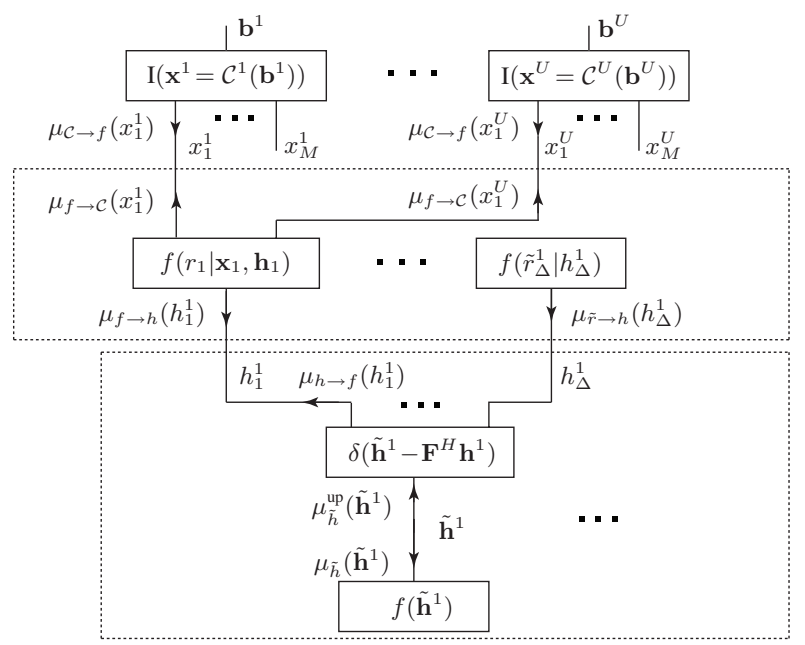

Fig. 2. Forney-style factor graph describing $f(\mathbf{X}, \mathbf{H}, \mathbf{r} \mid \mathbf{b})$ in (6) for the first symbol and one pilot symbol of the first user.

$$
p\left(b_{i}^{u} \mid \mathbf{r}\right)=\sum_{\sim b_{i}^{u}} p(\mathbf{b} \mid \mathbf{r}) \propto \sum_{\sim b_{i}^{u}} f(\mathbf{r} \mid \mathbf{b}),
$$

where $f(\mathbf{r} \mid \mathbf{b})$ is the conditional probability density function (pdf) of $\mathbf{r}$ given $\mathbf{b}, \sum_{\sim x}$ denotes summation over all unknown variables in the summand except $x$, and $\propto$ denotes equality up to factors irrelevant to the maximization (3). Based on the one-to-one correspondence between $\mathbf{b}$ and $\mathbf{X} \triangleq\left(\mathbf{x}^{1} \cdots \mathbf{x}^{U}\right)$, we can factor $f(\mathbf{r} \mid \mathbf{b})$ as

$$
f(\mathbf{r} \mid \mathbf{b})=\sum_{\mathbf{X}} f(\mathbf{r} \mid \mathbf{X}) \prod_{u=1}^{U} \mathrm{I}\left(\mathbf{x}^{u}=\mathcal{C}^{u}\left(\mathbf{b}^{u}\right)\right),
$$

where the indicator function $\mathrm{I}(\cdot)$ is one if its argument is true and zero otherwise. We have $f(\mathbf{r} \mid \mathbf{X})=\int f(\mathbf{r} \mid \mathbf{X}, \mathbf{H}) f(\mathbf{H}) d \mathbf{H}$, where $\mathbf{H}$ is the $U \times N$ matrix of all $h_{n}^{u}$. Using (1), (2) and the definitions $\mathbf{x}_{n} \triangleq\left(x_{n}^{1} \cdots x_{n}^{U}\right)^{T}$ and $\mathbf{h}_{n} \triangleq\left(h_{n}^{1} \cdots h_{n}^{U}\right)^{T}$ yields

$$
f(\mathbf{r} \mid \mathbf{X}, \mathbf{H})=\prod_{n \notin \mathcal{P}} f\left(r_{n} \mid \mathbf{x}_{n}, \mathbf{h}_{n}\right) \prod_{u=1}^{U} \prod_{n \in \mathcal{P}^{u}} f\left(\tilde{r}_{n}^{u} \mid h_{n}^{u}\right) .
$$

Furthermore, using the independence of all channels and $\mathbf{h}^{u}=\mathbf{F} \tilde{\mathbf{h}}^{u}$, we have $f(\mathbf{H})=\prod_{u=1}^{U} \int \delta\left(\tilde{\mathbf{h}}^{u}-\mathbf{F}^{H} \mathbf{h}^{u}\right) f\left(\tilde{\mathbf{h}}^{u}\right) d \tilde{\mathbf{h}}^{u}$. Combining these expressions and inserting them into (4), we obtain

$$
p\left(b_{i}^{u} \mid \mathbf{r}\right) \propto \sum_{\sim b_{i}^{u}} \int f(\mathbf{X}, \mathbf{H}, \mathbf{r} \mid \mathbf{b}) d \mathbf{H}
$$

with

$$
\begin{aligned}
f(\mathbf{X}, \mathbf{H}, \mathbf{r} \mid \mathbf{b})= & \prod_{n \notin \mathcal{P}} f\left(r_{n} \mid \mathbf{x}_{n}, \mathbf{h}_{n}\right) \prod_{u=1}^{U} \prod_{n \in \mathcal{P}^{u}} f\left(\tilde{r}_{n}^{u} \mid h_{n}^{u}\right) \\
& \times \prod_{u^{\prime}=1}^{U} \int \delta\left(\tilde{\mathbf{h}}^{u^{\prime}}-\mathbf{F}^{H} \mathbf{h}^{u^{\prime}}\right) f\left(\tilde{\mathbf{h}}^{u^{\prime}}\right) d \tilde{\mathbf{h}}^{u^{\prime}} \\
& \times \prod_{u^{\prime \prime}=1}^{U} \mathrm{I}\left(\mathbf{x}^{u^{\prime \prime}}=\mathcal{C}^{u^{\prime \prime}}\left(\mathbf{b}^{u^{\prime \prime}}\right)\right) .
\end{aligned}
$$

Due to (1) and (2), $f\left(r_{n} \mid \mathbf{x}_{n}, \mathbf{h}_{n}\right)$ and $f\left(\tilde{r}_{n}^{u} \mid h_{n}^{u}\right)$ are Gaussian with mean $\mathbf{h}_{n}^{T} \mathbf{x}_{n}$ and $h_{n}^{u} p_{n}^{u}$, respectively and variance $\sigma_{w}^{2}$. Fig. 2 depicts the Forney-style factor graph [6] corresponding to $f(\mathbf{X}, \mathbf{H}, \mathbf{r} \mid \mathbf{b})$ in

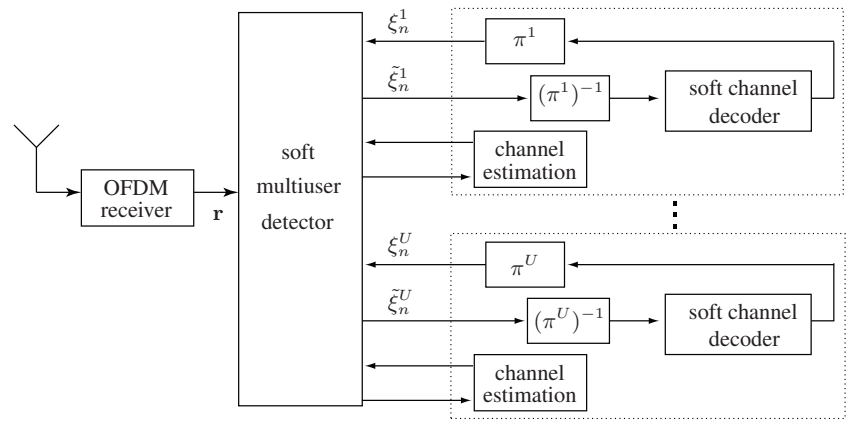

Fig. 3. Structure of the OFDM-IDMA receiver performing joint detection and channel estimation. ( $\xi_{n}^{u}$ and $\tilde{\xi}_{n}^{u}$ are defined in Section 4.)

(6). For simplicity, only the first symbol and one pilot symbol are shown.

Receiver structure. Applying the sum-product algorithm [5] to the factor graph in Fig. 2 yields an approximation to the marginal (5) for all $b_{i}^{u}$ simultaneously. (This is only an approximation because the graph contains cycles.) Using parallel scheduling [8], we obtain the receiver structure shown in Fig. 3. The block termed "soft multiuser detector" (corresponding to the upper dotted box in Fig. 2) receives soft information from the channel decoders of the individual users [1] and updates it using the current channel estimate. The improved soft bits are sent back to the decoders and also passed to channel estimation units (corresponding to the lower dotted box in Fig. 2), which calculate refined estimates of the channel coefficients. The decoding units-each consisting of deinterleaver, soft channel decoder, and interleaver-correspond to the blocks $\mathrm{I}\left(\mathbf{x}^{u}=\mathcal{C}^{u}\left(\mathbf{b}^{u}\right)\right)$ in Fig. 2. Upon termination of the sum-product algorithm, bit decisions are provided by the signs of the a posteriori soft information bits computed by the channel decoder.

\section{MESSAGES}

We now derive the messages that are propagated through the factor graph in Fig. 2.

Messages $\mu_{\mathcal{C} \rightarrow f}\left(x_{n}^{u}\right)$. Applying the sum-product algorithm to the code function nodes $\mathrm{I}\left(\mathbf{x}^{u}=\mathcal{C}^{u}\left(\mathbf{b}^{u}\right)\right)$ yields the BCJR algorithm $[5,9]$ for soft-decoding the convolutional code and a summation of appropriate bit log-likelihood ratios (LLRs) for soft-decoding the repetition code. This soft decoder produces extrinsic LLRs $\xi_{n}^{u} \in \mathbb{R}$ for the BPSK symbols $x_{n}^{u}$. The corresponding messages are [5]

$$
\mu_{\mathcal{C} \rightarrow f}\left(x_{n}^{u}\right)=\frac{\exp \left(\xi_{n}^{u}\left(x_{n}^{u}+1\right) / 2\right)}{1+\exp \left(\xi_{n}^{u}\right)}, \quad x_{n}^{u} \in\{-1,1\} .
$$

Messages $\mu_{f \rightarrow \mathcal{C}}\left(x_{n}^{u}\right)$. The channel function nodes pass the following messages back to the code function nodes:

$$
\begin{array}{r}
\mu_{f \rightarrow \mathcal{C}}\left(x_{n}^{u}\right)=\sum_{\sim x_{n}^{u}} \int f\left(r_{n} \mid \mathbf{x}_{n}, \mathbf{h}_{n}\right) \prod_{u^{\prime}=1}^{U} \mu_{h \rightarrow f}\left(h_{n}^{u^{\prime}}\right) \\
\times \prod_{u^{\prime \prime} \neq u} \mu_{\mathcal{C} \rightarrow f}\left(x_{n}^{u^{\prime \prime}}\right) d \mathbf{h}_{n} .
\end{array}
$$

The sum $\sum_{\sim x_{n}^{u}}$ is over $2^{U-1}$ terms. To achieve a complexity that is linear in $U$, we approximate the $\mu_{\mathcal{C} \rightarrow f}\left(x_{n}^{u}\right)$ by Gaussian messages,

$$
\mu_{\mathcal{C} \rightarrow f}\left(x_{n}^{u}\right)=\exp \left(-\frac{\left(x_{n}^{u}-a_{n}^{u}\right)^{2}}{2 b_{n}^{u}}\right),
$$


with mean $a_{n}^{u}=\tanh \left(\xi_{n}^{u}\right)$ and variance $b_{n}^{u}=1-\left(a_{n}^{u}\right)^{2}$ (cf. [1]). A Gaussian model is also used for the messages $\mu_{h \rightarrow f}\left(h_{n}^{u}\right)$, i.e.,

$$
\mu_{h \rightarrow f}\left(h_{n}^{u}\right)=\exp \left(-\frac{\left|h_{n}^{u}-\alpha_{n}^{u}\right|^{2}}{\beta_{n}^{u}}\right),
$$

where the mean $\alpha_{n}^{u}$ and variance $\beta_{n}^{u}$ will be determined later.

Assuming that $x_{n}^{u}$ and $h_{n}^{u}$ are independent and distributed according to (8) and (9), respectively, the message $\mu_{f \rightarrow \mathcal{C}}\left(x_{n}^{u}\right)$ in (7) equals the conditional pdf $f\left(r_{n} \mid x_{n}^{u}\right)$. Since closed-form integration in (7) is impossible, we use the Gaussian approximation

$$
\mu_{f \rightarrow \mathcal{C}}\left(x_{n}^{u}\right)=f_{\mathrm{G}}\left(r_{n} \mid x_{n}^{u}\right) \propto \exp \left(-\frac{\left|r_{n}-c_{n}^{u}\right|^{2}}{d_{n}^{u}}\right),
$$

with mean $c_{n}^{u}=\mathrm{E}\left\{r_{n} \mid x_{n}^{u}\right\}=\alpha_{n}^{u} x_{n}^{u}+\sum_{u^{\prime} \neq u} \alpha_{n}^{u^{\prime}} a_{n}^{u^{\prime}}$ and variance $d_{n}^{u}=\mathrm{E}\left\{\left|r_{n}-c_{n}^{u}\right|^{2} \mid x_{n}^{u}\right\}=\left|\alpha_{n}^{u}\right|^{2}+\sigma_{w}^{2}+\sum_{u^{\prime} \neq u}\left(\beta_{n}^{u^{\prime}}+\left|\alpha_{n}^{u^{\prime}}\right|^{2} b_{n}^{u^{\prime}}\right)$. In the course of the iterations, $b_{n}^{u} \rightarrow 0$ and $\beta_{n}^{u} \rightarrow 0$, i.e., $\mu_{\mathcal{C} \rightarrow f}\left(x_{n}^{u}\right)$ in (8) and $\mu_{h \rightarrow f}\left(h_{n}^{u}\right)$ in (9) that enter as factors in (7) become increasingly narrow, and thus the Gaussian approximation becomes more accurate. Converting $\mu_{f \rightarrow \mathcal{C}}\left(x_{n}^{u}\right)$ into an LLR value yields

$$
\tilde{\xi}_{n}^{u}=\log \frac{\mu_{f \rightarrow \mathcal{C}}\left(x_{n}^{u}=1\right)}{\mu_{f \rightarrow \mathcal{C}}\left(x_{n}^{u}=-1\right)}=\frac{\left(r_{n}-\sum_{u^{\prime} \neq u} \alpha_{n}^{u^{\prime}} a_{n}^{u^{\prime}}\right)\left(\alpha_{n}^{u}\right)^{*}}{2 d_{n}^{u}},
$$

which is passed to the associated channel decoder (see Fig. 3).

Messages $\boldsymbol{\mu}_{\boldsymbol{f} \rightarrow \boldsymbol{h}}\left(\boldsymbol{h}_{n}^{u}\right)$. The messages from the channel function nodes to the channel variable nodes are

$$
\begin{aligned}
\mu_{f \rightarrow h}\left(h_{n}^{u}\right)=\sum_{\mathbf{x}_{n}} \int f\left(r_{n} \mid \mathbf{x}_{n}, \mathbf{h}_{n}\right) \prod_{u^{\prime}=1}^{U} \mu_{\mathcal{C} \rightarrow f}\left(x_{n}^{u^{\prime}}\right) \\
\times \prod_{u^{\prime \prime} \neq u} \mu_{h \rightarrow f}\left(h_{n}^{u^{\prime \prime}}\right) d \mathbf{h}_{n}^{\sim u},
\end{aligned}
$$

where $\int d \mathbf{h}_{n}^{\sim u}$ denotes integration with respect to all entries of $\mathbf{h}_{n}$ except $h_{n}^{u}$. Based on arguments similar to those motivating (10), we use the approximation

$$
\mu_{f \rightarrow h}\left(h_{n}^{u}\right)=\exp \left(-\frac{\left|h_{n}^{u}-\nu_{n}^{u}\right|^{2}}{\gamma_{n}^{u}}\right)
$$

with $\nu_{n}^{u}=\frac{1}{a_{n}^{u}}\left(r_{n}-\sum_{u^{\prime} \neq u} \alpha_{n}^{u^{\prime}} a_{n}^{u^{\prime}}\right)$ and $\gamma_{n}^{u}=\frac{1}{\left|a_{n}^{u}\right|^{2}}\left[\sigma_{w}^{2}+b_{n}^{u}\left|h_{n}^{u}\right|^{2}\right.$ $\left.+\sum_{u^{\prime} \neq u}\left(\beta_{n}^{u^{\prime}}+\left|\alpha_{n}^{u^{\prime}}\right|^{2} b_{n}^{u^{\prime}}\right)\right]$. Because $h_{n}^{u}$ appears in $\gamma_{n}^{u}, \mu_{f \rightarrow h}\left(h_{n}^{u}\right)$ in (11) is not Gaussian in $h_{n}^{u}$. We thus use the approximation $\gamma_{n}^{u} \approx$ $\frac{1}{\left|a_{n}^{u}\right|^{2}}\left[\sigma_{w}^{2}+\sum_{u^{\prime} \neq u}\left(\beta_{n}^{u^{\prime}}+\left|\alpha_{n}^{u^{\prime}}\right|^{2} b_{n}^{u^{\prime}}\right)\right]$, which is justified because $b_{n}^{u} \rightarrow 0$ in the course of the iterations.

Messages $\boldsymbol{\mu}_{\tilde{r} \rightarrow \boldsymbol{h}}\left(\boldsymbol{h}_{n}^{u}\right)$. The messages from the pilot symbol function nodes are given by $\mu_{\tilde{r} \rightarrow h}\left(h_{n}^{u}\right)=\exp \left(-\left|\tilde{r}_{n}^{u}-h_{n}^{u} p_{n}^{u}\right|^{2} / \sigma_{w}^{2}\right)$. These messages can be rewritten as Gaussians in $h_{n}^{u}$ :

$$
\mu_{\tilde{r} \rightarrow h}\left(h_{n}^{u}\right)=\exp \left(-\frac{\left|h_{n}^{u}-\tilde{r}_{n}^{u} / p_{n}^{u}\right|^{2}}{\sigma_{w}^{2} /\left(p_{n}^{u}\right)^{2}}\right) .
$$

Messages $\mu_{\tilde{h}}\left(\tilde{\mathbf{h}}^{u}\right)$. Let us combine the (Gaussian) messages $\mu_{\tilde{r} \rightarrow h}\left(h_{n}^{u}\right)$ and $\mu_{f \rightarrow h}\left(h_{n}^{u}\right)$ into a "vector message" $\mu_{h}\left(\mathbf{h}^{u}\right)$, which is Gaussian with mean $\mathbf{m}_{h}^{u}$ and diagonal covariance $\mathbf{C}_{h}^{u}$. In the first several iterations, we use only the pilot symbols for channel estimation. We thus have $\mu_{h}\left(\mathbf{h}^{u}\right)=\prod_{n \in \mathcal{P} u} \mu_{\tilde{r} \rightarrow h}\left(h_{n}^{u}\right)$, from which it follows that $\left[\mathbf{m}_{h}^{u}\right]_{n}$ is $\tilde{r}_{n}^{u} / p_{n}^{u}$ if $n \in \mathcal{P}^{u}$ and zero otherwise, and $\left[\mathbf{C}_{h}^{u}\right]_{n, n}$ is $\sigma_{w}^{2} /\left(p_{n}^{u}\right)^{2}$ if $n \in \mathcal{P}^{u}$ and zero otherwise. In later iterations, we also use the messages $\mu_{f \rightarrow h}\left(h_{n}^{u}\right)$ for channel estimation, so $\mu_{h}\left(\mathbf{h}^{u}\right)=$ $\prod_{n \in \mathcal{P}^{u}} \mu_{\tilde{r} \rightarrow h}\left(h_{n}^{u}\right) \prod_{n \notin \mathcal{P}} \mu_{f \rightarrow h}\left(h_{n}^{u}\right)$. Thus, $\left[\mathbf{m}_{h}^{u}\right]_{n}$ is $\tilde{r}_{n}^{u} / p_{n}^{u}$ if $n \in$ $\mathcal{P}^{u}, \alpha_{n}^{u}$ if $n \notin \mathcal{P}$, and zero otherwise, and $\left[\mathbf{C}_{h}^{u}\right]_{n, n}$ is $\sigma_{w}^{2} /\left(p_{n}^{u}\right)^{2}$ if $n \in \mathcal{P}^{u}, \beta_{n}^{u}$ if $n \notin \mathcal{P}$, and zero otherwise. Finally, because $\tilde{\mathbf{h}}^{u}=$
$\mathbf{F}^{H} \mathbf{h}^{u}$, the desired message $\mu_{\tilde{h}}\left(\tilde{\mathbf{h}}^{u}\right)$ is Gaussian with mean $\mathbf{m}_{\tilde{h}}^{u}=$ $\mathbf{F}^{H} \mathbf{m}_{h}^{u}$ and covariance $\mathbf{C}_{\tilde{h}}^{u}=\mathbf{F}^{H} \mathbf{C}_{h}^{u} \mathbf{F}$.

Messages $\boldsymbol{\mu}_{\tilde{\boldsymbol{h}}}^{\text {up }}\left(\tilde{\mathbf{h}}^{u}\right)$. The message $\mu_{\tilde{h}}\left(\tilde{\mathbf{h}}^{u}\right)$ is multiplied by the $a$ priori message $\mu_{f_{\tilde{h}}}\left(\tilde{\mathbf{h}}^{u}\right)$ (corresponding to $f\left(\tilde{\mathbf{h}}^{u}\right)$ ), which is Gaussian with mean $\mathbf{m}_{\tilde{h}_{p}}$ and covariance $\mathbf{C}_{\tilde{h}_{p}}$. The product $\mu_{\tilde{h}}^{\text {up }}\left(\tilde{\mathbf{h}}^{u}\right)$ is

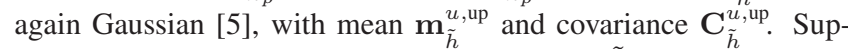
pose that the receiver uses a fixed channel length $\tilde{L}_{c}$ (in Section 5, we propose a method for estimating the channel length). Then, only the first $\tilde{L}_{c}$ entries of $\mathbf{m}_{\tilde{h}}^{u \text {,up }}$ (denoted by $\mathbf{n}_{\tilde{h}}^{u \text {,up }}$ ) and the top-left $\tilde{L}_{c} \times \tilde{L}_{c}$ submatrix of $\mathbf{C}_{\tilde{h}}^{u \text {,up }}$ (denoted $\mathbf{D}_{\tilde{h}}^{u \text {,up }}$ ) are nonzero. Let $\mathbf{n}_{\tilde{h}}^{u}$ and $\mathbf{n}_{\tilde{h}_{p}}$ denote the first $\tilde{L}_{c}$ entries of $\mathbf{m}_{\tilde{h}}^{u}$ and $\mathbf{m}_{\tilde{h}_{p}}$, respectively, and let $\mathbf{D}_{\tilde{h}}^{u}$ and $\mathbf{D}_{\tilde{h}_{p}}$ denote the top-left $\tilde{L}_{c} \times \tilde{L}_{c}$ submatrix of $\mathbf{C}_{\tilde{h}}^{u}$ and $\mathbf{C}_{\tilde{h}_{p}}$, respectively. Then $\mathbf{D}_{\tilde{h}}^{u \text {,up }}=\left(\left(\mathbf{D}_{\tilde{h}}^{u}\right)^{-1}+\mathbf{D}_{\tilde{h}_{p}}^{-1}\right)^{-1}$ and $\mathbf{n}_{\tilde{h}}^{u \text {,up }} \stackrel{\mathbf{h}_{p}}{=}$ $\mathbf{D}_{\tilde{h}}^{u \text {,up }}\left(\left(\mathbf{D}_{\tilde{h}}^{u}\right)^{-1} \mathbf{n}_{\tilde{h}}^{u}+\mathbf{D}_{\tilde{h}_{p}}^{-1} \mathbf{n}_{\tilde{h}_{p}}\right)[5]$.

Messages $\boldsymbol{\mu}_{\boldsymbol{h} \rightarrow \boldsymbol{f}}\left(\mathbf{h}^{u}\right)$. Because $\mathbf{h}^{u}=\mathbf{F} \tilde{\mathbf{h}}^{u}$, the message $\mu_{h \rightarrow f}\left(\mathbf{h}^{u}\right)$ (the "vector message" combining the messages $\mu_{h \rightarrow f}\left(\tilde{h}_{n}^{u}\right)$ ) is Gaussian with mean $\mathbf{m}_{h}^{u \text {,up }}=\mathbf{F} \mathbf{m}_{\tilde{h}}^{u \text {, up }}$ and covariance $\mathbf{C}_{h}^{u \text {,up }}=\mathbf{F} \mathbf{C}_{\tilde{h}}^{u, \text { up }} \mathbf{F}^{H}$. The mean $\alpha_{n}^{u}$ and variance $\beta_{n}^{u}$ in (9) equal the $n$th element of $\mathbf{m}_{h}^{u \text { up }}$ and the $n$th diagonal element of $\mathbf{C}_{h}^{u \text {,up }}$, respectively.

Scheduling. The messages $\mu_{f \rightarrow \mathcal{C}}\left(x_{n}^{u}\right)$ for all users at the input of the multiuser detector are simultaneously updated by the channel decoders and used by the multiuser detector to calculate the messages for all users at its output (parallel message scheduling [8]). We propose not to update the messages $\mu_{h \rightarrow f}\left(h_{n}^{u}\right)$ (using the messages $\left.\mu_{f \rightarrow h}\left(h_{n}^{u}\right)\right)$ during the first three iterations. Initially, a maximum value of $\tilde{L}_{c}$ is used; an estimate of $L_{c}$ is calculated (see Section 5) in the fifth iteration and then used for $\tilde{L}_{c}$ in all subsequent iterations.

\section{CHANNEL LENGTH ESTIMATION}

Estimating the channel length $L_{c}$ can improve receiver performance, because fewer channel coefficients have to be estimated. We consider the maximum likelihood estimator

$$
\hat{L}_{c}=\arg \max _{L_{c} \in \mathbb{N}} f\left(\mathbf{r} \mid L_{c}\right),
$$

where $f\left(\mathbf{r} \mid L_{c}\right)$ can be obtained by the following marginalization:

$$
f\left(\mathbf{r} \mid L_{c}\right) \propto \sum_{\mathbf{X}, \mathbf{b}} \int f\left(\mathbf{X}, \mathbf{H}, \mathbf{r} \mid \mathbf{b}, L_{c}\right) d \mathbf{H} .
$$

We have $f\left(\mathbf{X}, \mathbf{H}, \mathbf{r} \mid \mathbf{b}, L_{c}\right)=f\left(\mathbf{r} \mid \mathbf{X}, \mathbf{H}, \mathbf{b}, L_{c}\right) f\left(\mathbf{X}, \mathbf{H} \mid \mathbf{b}, L_{c}\right)=$ $f(\mathbf{r} \mid \mathbf{X}, \mathbf{H}) f(\mathbf{X} \mid \mathbf{b}) f\left(\mathbf{H} \mid L_{c}\right)$, with

$$
f\left(\mathbf{H} \mid L_{c}\right)=\prod_{u=1}^{U} \int \delta\left(\tilde{\mathbf{h}}^{u}-\mathbf{F}^{H} \mathbf{h}^{u}\right) f\left(\tilde{\mathbf{h}}^{u} \mid L_{c}\right) d \tilde{\mathbf{h}}^{u} .
$$

The marginalization (13) equals (5), except that the summation is over all entries of $\mathbf{b}$. Using the sum-product algorithm, we pass messages analogous to those used for approximating (5), except that now they depend on $L_{c}$. For a given $L_{c}$, the messages $\mu_{\tilde{h}}\left(\tilde{\mathbf{h}}^{u}\right)$ are multiplied by the a priori messages $\mu_{f_{\tilde{h}}}\left(\tilde{\mathbf{h}}^{u} \mid L_{c}\right)$ (formerly $\mu_{f_{\tilde{h}}}\left(\tilde{\mathbf{h}}^{u}\right)$ ), yielding $\mu_{\tilde{h}}^{\text {up }}\left(\tilde{\mathbf{h}}^{u} \mid L_{c}\right)$. From these, the messages $\mu_{h \rightarrow f}\left(h_{n}^{u} \mid L_{c}\right)$ are obtained as in Section 4, and (13) can be expressed as

$$
\begin{aligned}
f\left(\mathbf{r} \mid L_{c}\right) \propto \prod_{n \notin \mathcal{P}} \int f\left(r_{n} \mid \mathbf{x}_{n},\right. & \left.\mathbf{h}_{n}\right) \prod_{u=1}^{U} \mu_{\mathcal{C} \rightarrow f}\left(x_{n}^{u}\right) \\
& \times \mu_{h \rightarrow f}\left(h_{n}^{u} \mid L_{c}\right) d x_{n}^{u} d h_{n}^{u} .
\end{aligned}
$$




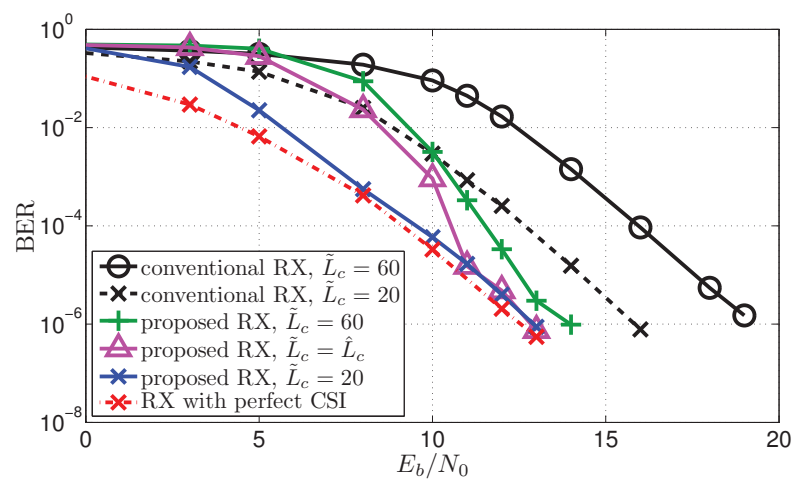

Fig. 4. Average BER versus $E_{b} / N_{0}$ obtained with different OFDMIDMA receivers for $U=4$ users and channel length $L_{c}=20$.

Using the approximation $\mu_{h \rightarrow f}\left(h_{n}^{u} \mid L_{c}\right)=\delta\left(h_{n}^{u}-\alpha_{n}^{u}\left(L_{c}\right)\right)$ (cf. (9)), the $h_{n}^{u}$ integral collapses. This yields

$$
f\left(\mathbf{r} \mid L_{c}\right) \propto \prod_{n \notin \mathcal{P}} \int f\left(r_{n} \mid \mathbf{x}_{n}, \mathbf{h}_{n}=\boldsymbol{\alpha}_{n}\left(L_{c}\right)\right) \prod_{u=1}^{U} \mu_{\mathcal{C} \rightarrow f}\left(x_{n}^{u}\right) d x_{n}^{u},
$$

with $\boldsymbol{\alpha}_{n}\left(L_{c}\right) \triangleq\left(\alpha_{n}^{1}\left(L_{c}\right) \cdots \alpha_{n}^{U}\left(L_{c}\right)\right)^{T}$. The $\mu_{\mathcal{C} \rightarrow f}\left(x_{n}^{u}\right)$ are Gaussian (see (8)), so the integration can be carried out and we obtain

$$
f\left(\mathbf{r} \mid L_{c}\right) \propto-\sum_{n \notin \mathcal{P}}\left|r_{n}-\sum_{u=1}^{U} a_{n}^{u} \alpha_{n}^{u}\left(L_{c}\right)\right|^{2} .
$$

The maximization (12) is then done by exhaustive search.

\section{SIMULATION RESULTS}

We consider an OFDM-IDMA system with $U=4$ users, each transmitting $K=256$ information bits. An overall code rate of $1 / 8$ is achieved by serially concatenating a terminated rate- $1 / 2$ convolutional code (code polynomial $\left[\begin{array}{ll}2 & 3\end{array}\right]_{8}$ ) and a rate- $1 / 4$ repetition code (hence, the sum-rate is $1 / 2$ ). $N=2360$ subcarriers are used, with a pilot spacing of $\Delta=30$. The a priori variances of the nonzero channel coefficients were set equal to 1 .

For a channel of length $L_{c}=20$, Fig. 4 shows the average bit error rate (BER) versus the signal-to-noise ratio (SNR) $E_{b} / N_{0}$ for the following receivers: (i) a conventional receiver that performs pilotbased MMSE channel estimation assuming $\tilde{L}_{c}=60$ and uses the resulting channel estimates for iterative data detection; (ii) the same receiver but assuming the correct channel length, i.e., $\tilde{L}_{c}=L_{c}=20$; (iii) the proposed receiver without channel length estimation, assuming $\tilde{L}_{c}=60$; (iv) the proposed receiver with channel length estimation; (v) the proposed receiver without channel length estimation but assuming $\tilde{L}_{c}=L_{c}=20$; and (vi) an iterative receiver with perfect channel state information (CSI). All receivers performed 10 iterations. The conventional receiver with $\tilde{L}_{c}=60$ is seen to perform about $3 \mathrm{~dB}$ worse than the conventional receiver assuming the correct $L_{c}$. At a BER of $10^{-6}$, the proposed receiver using channel length estimation and the proposed receiver using the true $L_{c}$ perform almost as well as the receiver with perfect CSI, while the proposed receiver using $\tilde{L}_{c}=60$ performs about $1 \mathrm{~dB}$ worse. These results demonstrate the advantages of joint data detection and channel estimation, as well as of channel length estimation, in the OFDM-IDMA context considered.

To illustrate the performance of the proposed channel length estimation method, Fig. 5 shows histograms of $\hat{L}_{c}$ for two channels with $L_{c}=20$ and $L_{c}=40$, at $E_{b} / N_{0}=11 \mathrm{~dB}$. It is seen that the estimates

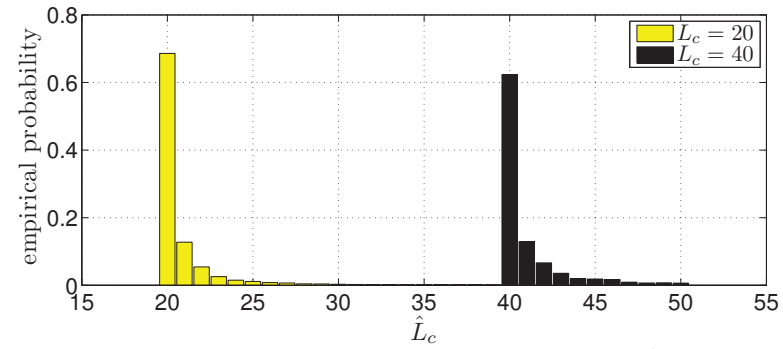

Fig. 5. Histogram of the estimated channel length $\hat{L}_{c}$ for channel lengths $L_{c}=20$ and $L_{c}=40$, at $E_{b} / N_{0}=11 \mathrm{~dB}$.

are correct in the majority of cases. There is a tendency for overestimation, but estimation errors larger than 2 are quite unlikely. We note that overestimation of $L_{c}$ causes a much smaller BER increase than underestimation.

\section{CONCLUSION}

Using a factor graph approach, we developed an iterative OFDMIDMA receiver whose complexity scales linearly with the number of users. Performance improvements relative to conventional receivers were achieved by joint multiuser data detection and channel estimation and by explicit estimation of the length of the frequencyselective channels. Extensions to MIMO-OFDM-IDMA systems with spatial multiplexing (cf. [10]) and to time-varying channels, as well as possible combination with reduced-rank approximations [11] are interesting directions for future research.

\section{REFERENCES}

[1] L. Ping, L. Liu, K. Wu, and W. K. Leung, "Interleave-division multipleaccess," IEEE Trans. Wireless Comm., vol. 5, pp. 938-947, Apr. 2006.

[2] I. Mahafeno, C. Langlais, and C. Jego, "OFDM-IDMA versus IDMA with ISI cancellation for quasi-static Rayleigh fading multipath channels," in Proc. 4th Int. Symp. on Turbo Codes \& Related Topics, (Munich, Germany), pp. 3-7, 2006.

[3] L. Ping, Q. Guo, and J. Tong, "The OFDM-IDMA approach to wireless communication systems," IEEE Wireless Comm., vol. 14, pp. 18-24, June 2007.

[4] C. Novak, G. Matz, and F. Hlawatsch, "A factor graph approach to joint iterative data detection and channel estimation in pilot-assisted IDMA transmissions," in Proc. IEEE ICASSP-2008, (Las Vegas, NV), pp. 2697-2700, Apr. 2008.

[5] H.-A. Loeliger, J. Dauwels, J. Hu, S. Korl, L. Ping, and F. R. Kschischang, "The factor graph approach to model-based signal processing," Proc. IEEE, vol. 95, pp. 1295-1322, June 2007.

[6] G. D. Forney, Jr., "Codes on graphs: Normal realizations," IEEE Trans. Inf. Theory, vol. 47, pp. 520-548, Feb. 2001.

[7] A. P. Worthen and W. E. Stark, "Unified design of iterative receivers using factor graphs," IEEE Trans. Inf. Theory, vol. 47, pp. 843-849, Feb. 2001.

[8] J. Boutros and G. Caire, "Iterative multiuser joint decoding: Unified framework and asymptotic analysis," IEEE Trans. Inf. Theory, vol. 48, pp. 1772-1793, July 2002.

[9] L. R. Bahl, J. Cocke, F. Jelinek, and J. Raviv, "Optimal decoding of linear codes for minimizing symbol error rate," IEEE Trans. Inf. Theory, vol. 20, pp. 284-287, March 1974.

[10] C. Novak, F. Hlawatsch, and G. Matz, "MIMO-IDMA: Uplink multiuser MIMO communications using interleave-division multiple access and low-complexity iterative receivers," in Proc. IEEE ICASSP2007, (Honolulu, Hawaii, USA), pp. 225-228, Apr. 2007.

[11] K. Kusume, G. Dietl, W. Utschick, and G. Bauch, "Performance of interleave division multiple access based on minimum mean square error detection," in Proc. IEEE ICC-2007, (Glasgow, UK), pp. 2961-2966, June 2007. 